\title{
Hot Melt Extrusion an Emerging Pharmaceutical Technology
}

\author{
Sagar Kothawade ${ }^{1 *}$, Rutuja Wakure ${ }^{1}$, Shubham Biyani ${ }^{2}$, Vijay Thalapally ${ }^{2}$, Bhagwan Bukya ${ }^{1}$
}

${ }^{1}$ Murlikrishna Pharmaceutical Pvt. Ltd, MIDC Ranjangaon, Maharashtra 412209,, India

${ }^{2}$ Aurobindo Pharma Research Centre II, Sanga Readdy, Telangana 502329, India

DOI: $10.36347 /$ sajp.2020.v09i06.002

| Received: 30.04.2020 | Accepted: 08.05.2020 | Published: 06.06.2020

*Corresponding author: Sagar Kothawade

Hot Melt Extrusion technology offers an opportunity to acquire intellectual property, evident from the increasing number of patents and publications that have integrated it as an emerging pharmaceutical formulation technology over the last decades. Industrial adaptability has allowed hot-melt extrusion (HME) to gain wide recognition over the last decades and has already established its place in a wide range of manufacturing operations and advancements in pharmaceutical research. This study addressed the importance of the use of a quality-based strategy, based on a holistic approach to equipment, materials processing methods, novel formulation design and advancements, and its various applications for oral drug delivery systems. In drug discovery and development, the industry estimates that more than $50 \%$ of the active pharmaceutical ingredient used is part of the Biopharmaceutical Classification System II, defined as water-insoluble compounds, leading to formulations having less bioavailability. The pharmaceutical industry needs to develop formulations that will improve the solubility and ultimately the bioavailability of these compounds. Hot Melt Extrusion has already been shown to be robust, novel and powerful technique for improved bioavailability as well as taste masking of bitter active pharmaceutical ingredients (APIs). This paper examines the many benefits of Hot Melt Extrusion, based on a holistic approach to equipment, material processing methods, novel design and advancements.

Keywords: Hot Melt Extrusion, Solid-dispersion, Bioavailability, Application.

Copyright @ 2020: This is an open-access article distributed under the terms of the Creative Commons Attribution license which permits unrestricted use, distribution, and reproduction in any medium for non-commercial use (NonCommercial, or CC-BY-NC) provided the original author and source are credited.

\section{INTRODUCTION}

Extrusion is a process in which the homogeneous product is formed by converting the powder into a molten mass through accurate control of the processing condition. Hot Melt Extrusion (HME) has emerged as a novel processing technique in the development of molecular dispersion through various polymer or lipid matrices of active pharmaceutical ingredients, resulting in time-controlled, prolonged and targeted drug delivery [1-3]. HME has now provided the opportunity to use materials to mask the bitter taste and enhance the solubility of active substances. In 1797 Joseph Bramah patented the first extrusion process for the production of soft metal pipes [4]. It involved preheating the metal and then forcing it through a die through a hand-driven plunger. In 1820, Thomas Burr carried out this process for the hydraulic press lead pipe (also invented by Joseph Bramah) and at that time the process was called "squirting." In 1894, Alexander Dick extended the process of extrusion to copper and brass alloys $[5,6]$.

In the 1930s, the industrial application of the extrusion process quickly became the most widely used processing technology in the plastics, rubber and food industries [7, 8]. The application of extrusion expanded to the pharmaceutical industry at the beginning of the 1970 s and was used in formulation and product development as well as manufacturing. HME technology was firstly introduced into the pharmaceutical industry in 1991 when Warner-Lambert used HME to enhance the solubility of Troglitazone which is very poorly soluble drug $[9,10]$. The company had bought an early phase medication product Troglitazone two years ago, utilizing a spray drying method to improve drug material solubility by the creation of amorphous solid dispersion. HME has received considerable attention from both the pharmaceutical industry and academics to the development of pharmaceutical dosage forms such as tablets, capsules, film and drug delivery implants via oral, transdermal and Trans muscular routes [11, 12]. This makes the HME an ideal alternative to other conventionally accessible roll spinning and spray drying techniques. HME also fulfills the U.S. Food and Drug Administration's mission, which is focused on a comprehensive view of its multiple elements, manufacturing techniques and resources, and the 
innovative nature and creation of new formulations in its complex use in the oral drug delivery system [13, 14]. As part of the development of the pharmaceutical formulation, HME has made available sophisticated equipment such as customized downstream auxiliary extruders and monitoring tools for use in performance and product quality assessments. This increased availability has supported the growth of this innovative technology in pharmaceutical manufacturing processes using single and twin-screw extruders [15]. The approved formulation in market by hot melt extrusion proves shown in Table No I.

Table-I: Marketed formulation by hot melt extrusion

\begin{tabular}{|l|l|l|l|}
\hline Brand Name & Approved Year & Product & Types of System \\
\hline Merck & 1981 & Lacrisert & Shaped Systems \\
\hline Pifzer & 1996 & Zothromax & Sustained Release \\
\hline Merck & 2001 & Nuva Ring & Shaped System \\
\hline Purdue Pharma & 2004 & Palladoone & Sustained Release \\
\hline Abb Vie & 2005 & Kaletra & Immediate Release \\
\hline Merck & 2006 & Implanon & Shaped system \\
\hline Novartis & 2007 & Eucreas & Immediate Release \\
\hline Allegran & 2009 & Ozurdex & Shaped System \\
\hline Abb Vie & 2010 & Nucynta & Sustained Release \\
\hline Merck & 2013 & Noxafil & Delayed Release \\
\hline Merck & 2014 & Belspmra & Immediate Release \\
\hline Merck & 2015 & Zepatier & Immediate Release \\
\hline
\end{tabular}

\section{Equipment and processing of hot melt extrusion}

In Extrusion process, the drug is mixed with a suitable polymer to form the molten mass by forcing it through a heated barrel or die under controlled temperature and pressure. The Extruders are classified into three main categories Ram, Radial screen and Screwed extruder [16]. Among these, the screws Extruders in the pharmaceutical industry are the most important as they continuously transform feed material to the finished shape such as a pipe, tube, or film. The spinning screws push the feed content against the die, and the frictional heat produced through the barrel wall softens the substance. The feed reaches the end of the screw in a viscous state and then forced through an orifice and molded into the desired shape [17] is the process of Hot melt extrusion shown in Fig No 1.

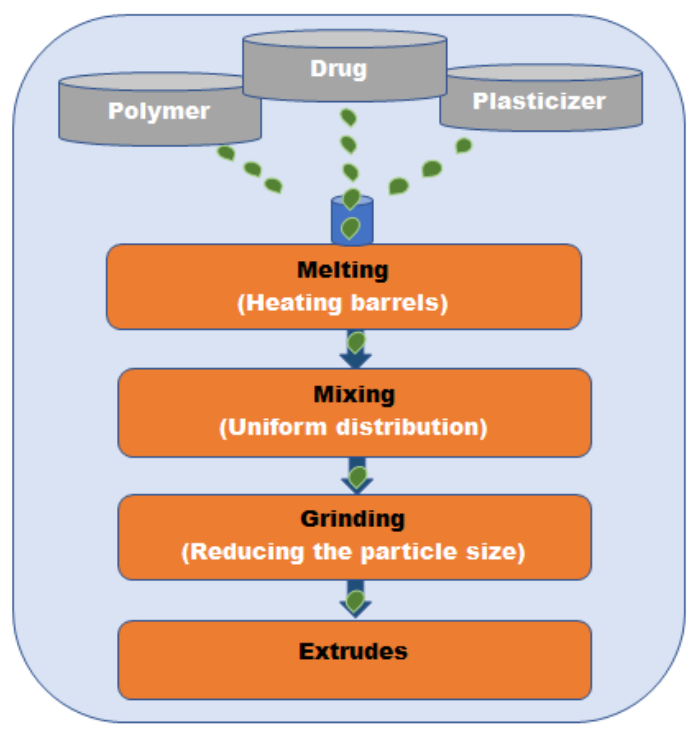

Fig-1: Hot melt extrusion process and design

\section{PARTS OF EXTRUDER Barrels}

Extruder barrels are the housing for the screws.

The barrels are generally electrically heated and water cooled. During the extrusion process the barrel temperature is monitored by thermocouples. Depending on the design and manufacturing the barrel can be either a one-piece or a modular design. Compared to the one- piece design modular barrels are more widely used in the pharmaceutical industry due to their greater flexibility [18].

\section{Different types of barrels [19]}

The different type of barrel is used in hot melt extrusion process are shown in Fig No 2. 


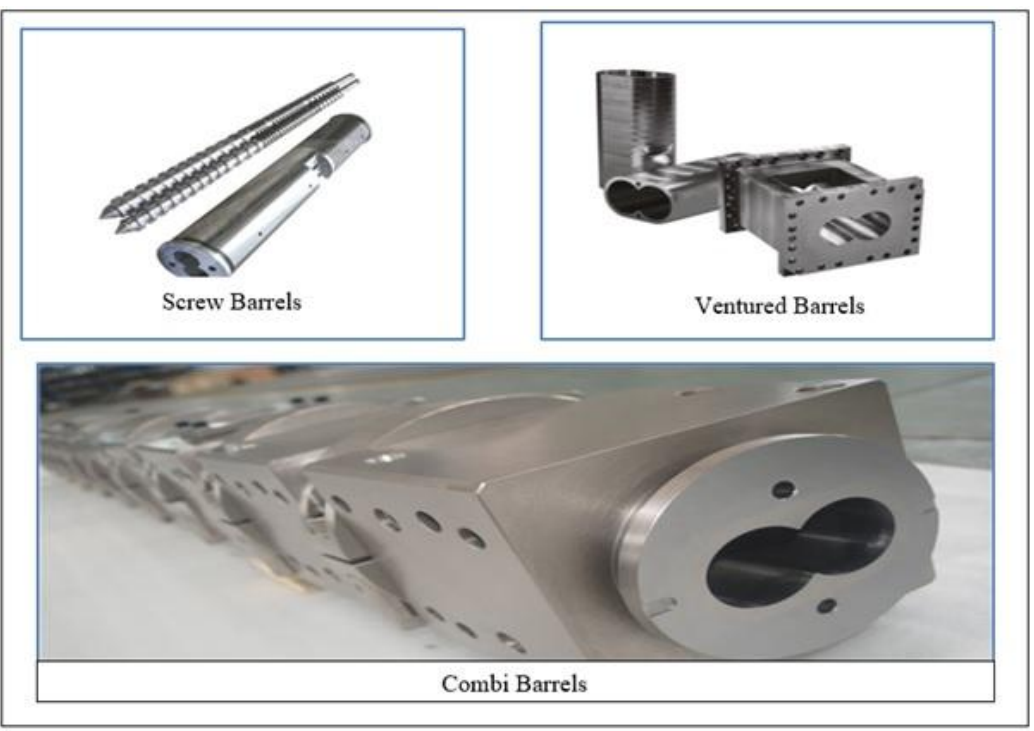

Fig-2: Different types barrels used in HME process

a) Closed barrel and combo: This type of barrels can be used for liquid feed. This type of barrels can be used for liquid feed. Used, if needed it can be introduced into the system through an injection nozzle connected to a closed barrel.

b) Feed barrel: It is either in a circular or rectangular shape. It normally kept at near to the room temperature to maximize the free volume of material to enter into the extruder.

c) Vented barrel: It is used to remove air and/or volatiles from the extruder. The process is also referred as devolatilization.

\section{Screw element}

Screw construction may have a major effect on the functionality and efficiency of the component as it impacts mechanical shear and period of residence. For a wet granulation process, Thompson and Sun stated that modifications in the screw elements may affect the size and shape of lactose-polyvinylpyrrolidone granules. Nakamichiet et al. showed that kneading paddles play an important role in the preparation of amorphous solid dispersion (ASD) of nifedipine. Therefore, the special attention should be given while designing the screws. During design the process requirements and the material properties must be considered. Screw configuration can be designed as shear-intensive or shear-passive according to process requirements [20].

\section{Dies}

The die opening is attached to end of the extruder and the pressurized molten mass is passed from the barrel and enters into the die. The main function of die is to convert the pressurized molten mass into desired shape and size.

\section{Auxiliary systems}

A wide range of auxiliary systems including feeders and various downstream equipment are used in the extrusion process. The selection of feeder and downstream equipment with extruder is very important for completion of the extrusion process [21].

\section{PROCESS PARAMETERS}

Extrusion is an integrated unit operation composed of several functional zones. Process parameter plays a key role in determining the properties of the extruded product. The complexity of the material and energy properties in different functional zones thought the extruder. The product quality is controlled directly by key system parameters such as specific energy, temperature, residence time and pressure. While the common adjustable equipment and process parameters impact the product quality indirectly. Key process considerations based on the adjustable parameter (e.g. Feed rate, screw speed, temperature profiles) Table No II [22].

Table-II: Process parameter of hot melt extrusion

\begin{tabular}{|l|l|l|l|l|l|l|}
\hline Function Zone & Slid feed & Slid convey & Melting & Mixing & $\begin{array}{l}\text { Melt } \\
\text { Convey }\end{array}$ & Discharge \\
\hline Material State & Solid & Solid & Slid/Melt & Melt & Melt & Melt \\
\hline Pressure (Bar) & 1 & 1 & 1 & $>1$ & $>1$ & $>1$ \\
\hline Conductive Energy & $\begin{array}{l}\text { Out of } \\
\text { Material }\end{array}$ & $\begin{array}{l}\text { In and out of } \\
\text { Material }\end{array}$ & Out of material & $\begin{array}{l}\text { Out of } \\
\text { Material }\end{array}$ & $\begin{array}{l}\text { Out of } \\
\text { Material }\end{array}$ & $\begin{array}{l}\text { Out of } \\
\text { Material }\end{array}$ \\
\hline Energy Conversion & None & None & PED/FED & VED & VED & VED \\
\hline
\end{tabular}




\section{MATERIALS USED IN HOT MELT EXTRUSION} Drug

HME can be conducted below the melting point of a drug that means the melting point of a drug is depressed when there is a strong interaction between the drug and the polymer. Intermolecular interaction between drug and polymer is important for the physical stability of amorphous solid dispersion (ASD). Interaction between drug and polymer can be determined by experimentation using the melting-point depression method. Good miscibility between drug polymers manifests itself as the depression of drug melting point. The Gibbs free energy of the dissolved drug in its saturated solution in polymer melts [23].

\section{Polymer}

Polyethyleneglycol-polyyvinyl caprolactampolyvinyl acetate grafted copolymer is a newly developed polymer commonly used in HME for drug development. The polymer has an amphiphilic structure that enables it to behave as both a polymer-forming matrix and a solubilizer for poorly soluble APIs in aqueous solutions. It has a low glass transition temperature of $70{ }^{0} \mathrm{C}$. Figure 3 shows the relationship between the glass transition temperature and the polymer state. Djuris et al. Have demonstrated with a single screw hot-melt extruder that a mixture of carbamazepine and Polyethyleneglycol-polyvinyal caprolactam-polyvinyl acetate thermodynamic model. In another example, the oral bioavailability of Valsartan was improved by melt extrusion with this copolymer and the addition of a non-ionic surfactant, Vitamin $\mathrm{E}$ TPGS [24]. Table No III shows the selection of polymers based on dosage form.

Table-III: Selection of Polymer in Hot Melt Extrusion

\begin{tabular}{|l|l|}
\hline Dosage form & Polymer \\
\hline Oral / Ingestible & $\begin{array}{l}\text { Polyvinyl pyrrolidone (PVP), Polyethylene oxide (PEO), Hydroxypropyl Cellulose (HPC), } \\
\text { Hydroxypropylmethylcellulose Acetate Succinate (HPMCAS), Ethyl cellulose (EC), Cellulose } \\
\text { acetate butyrate (CAB), Cellulose Acetate Phthalate (CAP), Polyvinyl alcohol (PVA), Poly } \\
\text { (ethylene glycol) (PEG), Poly (vinyl acetate) (PVAc), Methacrylate's. }\end{array}$ \\
\hline Bioresorbable & Polylactide (PLA), Polyglycolide (PGA), Copolymers of PLA/PGA and Polycaprolactone (PCL). \\
\hline Implantable & Ethylene Vinyl Acetate (EVA), Polyurethanes (TPU), Polyethylene (PE). \\
\hline
\end{tabular}

\section{Plasticizer}

For plastics and pharmaceutical industries, the application of a plasticizer to promote the cycle of hot melt-extrusion is well known. A plasticizer is a low molecular weight material which is capable of polymer softening, the polymer more robust along with elongation and strength. Plasticization of polymer is due to the inter molecular secondary valance force between plasticizer which leads to increase free volume between polymer chain and decrease in the glass transition temperature [25-30]. Table No IV shows the examples of plasticizers.

Table-IV: Example of Plasticizer

\begin{tabular}{|l|l|}
\hline Class & Examples \\
\hline Polyhydric Alcohols & Propylene glycol, Glycerol, Polyethylene glycols \\
\hline Acetate Esters & Glyceryl triacetate, Acetyl triethyl citrate \\
\hline Phthalate Esters & Diethyl Phthalate \\
\hline Glycerides & Acetylated Monoglycerides \\
\hline Oils & Castor oil, Mineral oil \\
\hline
\end{tabular}

Factors affecting the selection of plasticizer [31-34]

1. Efficiency: The quantity of plasticizer required to lower the glass transition temperature, $(\mathrm{Tg})$ and produce desired effects indicates the efficiency of plasticizer. The diffusion rate of plasticizer is directly proportional to efficiency shown in Fig No 3. 


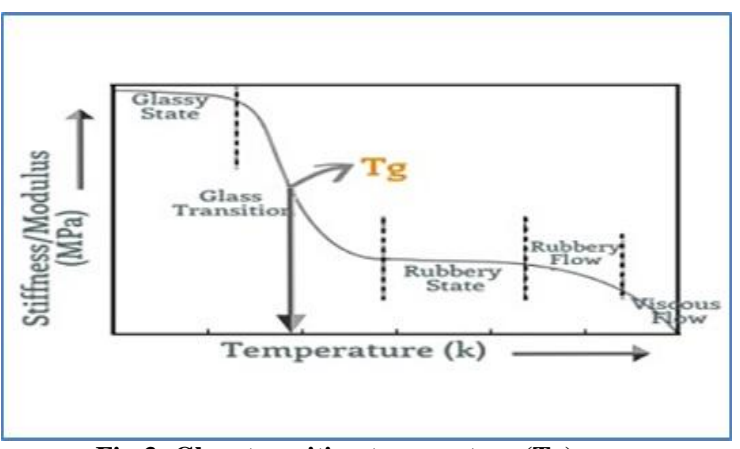

2. Compatibility: The compatibility of Plasticizer with polymer is depends on its phytochemical parameter such as Polarity, Structural configuration and Molecular weight of plasticizer. The compatibility of plasticizer is determined by the rule "like dissolved like" with the help of Hildebrand solubility and Hansen solubility determination methods.

3. Permanence: The permanence is a process of expulsion of plasticizer due to its higher volatility, which result plasticizer to come out of the polymer matrix. e. g. large alkyl moieties such as phthalates have increased hydrophobicity.

\section{Advantages of hot melt extrusion [35-40]}

HME has several benefits over conventionally accessible pharmaceutical processing methods.

1. Upgrades bioavailability of poorly soluble compound.

2. HME is economical, practical procedure with diminished creation time, less handling advances and a continued activity.

3. HME avoids dose dumping and clinically helpful in drug abuse.

4. HME can deliver drugs in a sustained manner and targeted drug delivery.

5. HME Produces various size ranges and geometries as well as show better content uniformity.

6. Compressibility of an active ingredient is not necessary.

7. HME process shows uniform dispersion of fine particles and also has good stability at varying $\mathrm{pH}$ and moisture level.

8. Reduced number of unit operations.

9. The process can be carried out in absence of solvents.

\section{Limitation of hot melt extrusion [41-43]}

Difficulties in maintaining stability for thermo sensitive drug and polymer

Required food flow property for polymer employed.

There are a limited number of polymers that used for HME.

The HME process requires high energy input.

\section{Pharamceutical applications of hot melt extrusion}

The initial research concentrated on the impact of the development and processing variables on attributes of the finished dosage form.

\section{Modified release formulation}

The sustained release of drug with combination of polymer formulated into solid dispersion by fusion method. e.g. In hot melt extrusion Sucrose esters are applied as processing carriers because of their low melting points and their surfactant properties. It was investigated by performing ibuprofen and sucrose ester used as promising carrier. Ibuprofen is a safe non-steroidal anti-inflammatory drug [44] and it has a short elimination half-life 2-3 hours, oral dose is 1.2 to $2.4 \mathrm{~g}$ daily in divided. This short half-life necessitates the frequent drug administration. Consequently, a sustained release dosage form would reduce the frequent drug administration and may enhance patient compliance [45].

\section{Targeted drug delivery system}

The transdermal and transmucosal dosage form like film and patch are produced more frequently by solvent casting method [46]. However, there are several problems with the solvent casting technique like physical aging effect on mechanical strength and increased film tensile strength [47]. This mechanical instability was related to the relaxation of the polymer chains as they moved towards a state of equilibrium. It directly affects the dissolution [48]. Rebekah et al. discussed the numerous disadvantages of solvent casting methods for preparing transdermal films [49]. Rothen-Weinhold developed long-acting pull (lactic acid) implants are comprised vapreotide, an equivalent somatostatin through extrusion from the hot melt. The authors reported that the peptide degraded during processing with the formation of a lactyl-vapreotide conjugate. The authors determined that residual lactide in the polylactic acid significantly influenced the formation of the peptide impurity, illustrating that carrier purity impacts the quality of the dosage form $[50,51]$.

\section{Improve solubility and bioavailability}

HME is recently used in healthcare industry to manufacture medical devices to enhance bioavailability of poorly soluble drug which belongs to BCS class II and IV. It has been successfully achieved through the formation of solid dispersion of drug in a polymeric 
carrier matrix. HME may be classified as solid crystalline dispersions, solid amorphous dispersions, and solid solutions. Amorphous material dispersion gives not only the intrinsic free energy advantages but also optimum surface area rises and solubility of saturation [52].

\section{Taste masking by HME approach}

It is a bigger challenge to mask the taste of bitter active pharmaceutical ingredients in formulation and development of oral dosage form. The taste masking of bitter drug can be achieved by HME via various mechanisms such as the formation of solid dispersions and inter-molecular interactions [53].

\section{Evaluation hot melt extrudes [54]}

Thermal and chemical stability of extrudes: Thermal transitions of the polymers are also indicative of the temperature of the extrusion. The glass transition temperatures and melting points were measured using a differential calorimeter. For stability testing of the polymers, three types of methods were considered, i.e. thermogravimetry, preliminary extrusion tests and highperformance liquid chromatography. With the first one the temperature profile weight loss was recorded using thermobalance.

Differential Scanning Calorimetry: DSC was widely used to study the thermal properties of the material used in HME. It can be used to determine the TG and Tm in which energy is required or released. It also used in drug excipient incompatibility studies. It was also used to distinguish between amorphous and crystalline forms.

Thermo Gravimetric Analysis: TGA is a measure of thermally caused material weight loss as a function of applied temperature. TGA is severely limited to, research involving either weight gain or loss and its frequency used for the research of desolvation and decomposition.

Infrared Spectroscopy: IR can be used to identify changes in linkages between functional groups due to structural modifications or absence of crystal structure.

\section{Physical state of extrudes}

XRD: It is used to characterize the crystalline characteristics of hot melt extruded dosage forms. The XRD principle is based on Bragg's in which parallel incident X-rays strike crystal surfaces and then diffract at angles linked to the spacing between planes of molecules in the lattice. Crystallinity is represented in the diffraction pattern by the distinctive fingerprint region. However, the sensitivity of the XRD technique is limited and cannot usually detect crystallinity of less than $10 \%$.
Microscopy: Is one of the best techniques for studying the crystalline characteristics of hot-melt extrudates. Both optical and electron techniques are appropriate for examining the surface morphology of the sample to be tested for the existence of crystalline particles or amorphous domains. Reliable data on particle size can also be obtained using these methods.

\section{CONCLUSION}

Hot Melt Extrusion has been well explored and documented as a steady, solvent-free, fast and costeffective manufacturing method for the production of a broad range of pharmaceutical dosage forms, including oral, topical film, implants and ophthalmic inserts. HME has demonstrated to be a robust method of producing a number of drug delivery system. There is increasing interest in HME technology as it is appropriate for both elevated and very powerful lowdose compounds, as well as the equipment is commonly accessible.

\section{Conflict of Intrest} of interest

All Authors declare that they have no conflict

\section{REFERENCES}

1. Bhagurkar A, Darji M, Lakhani P, Thipsay $P$, Bhandari S, Repka A. Effect of formulation composition on the characteristics of mucoadhesive films prepared by hot melt extrusion technology, J Pharm Pharmacol. 2019; 71(3): 293-305.

2. Chokshi R, Zia H. Hot Melt Extrusion Technique: A Review. Iranian Journal of Pharmaceutical Research. 2004; 3: 3-16.

3. Crowley MM, Zhang F, Repka MA, Thumma S, Upadhye SB, Battu SK, McGinity J, Martin C. Pharmaceutical Application of Hot-Melt Extrusion: Part I. Drug Dev Ind Pharm. 2007; 33: 909-926.

4. Dixit N, Maurya S, Sagar B. Sustained Release Drug Delivery System. Indian Journal of Research in Pharmacy and Biotechnology, 2013; 1(3): 305310.

5. M. Wilson, M. A. Williams, D. S. Jones, and G. P. Andrews, "Hot-melt extrusion technology and pharmaceutical application," Therapeutic Delivery. 2012; 3(6):787-797.

6. P. K. Mididoddi and M. A. Repka, "Characterization of hot-melt extruded drug delivery systems for onychomycosis," European Journal of Pharmaceutics and Biopharmaceutics. 2007; 66(1): 95-105.

7. Zhang F, McGinity JW. Properties of Sustained Release Tablets Prepared by Hot-Melt Extrusion. Pharmaceutical Development and Technology. 1999; 4(2): 241-250.

8. Foster A, Hempenstall J, Tucker I, Rades T. Selection of Excipients for Melt Extrusion with Two Poorly Water-Soluble Drugs by Solubility Parameter Calculation and Thermal Analysis. Int $\mathbf{J}$ Pharm. 2001; 226: 147-161. 
9. Brabander C, Vervaet C, Fiermans L, Remond JP. Matrix Mini-Tablets Based on Starch/Microcrystalline Wax Mixtrues, Int J Pharm. 2000; 199(2): 15-203.

10. Breitkreutz J, Saleh F, Kiera C, Kleinebudde P, Wiedey W. Paediatric Drug Formulations of Sodium Benzoate: Coated Granules with a Lipophilic Binder. Eur J Pharm Biopharm. 2003; 56 (2): 255-260.

11. Cilurzo F, Cupone I. E, Minghetti P, Selmin F, Montanari L. Fast Dissolving Films Made of Maltodextrins. Eur J Pharm Biopharm. 2008; 70(3): 895-900.

12. Crowley MM, Zhang F, Koleng JJ, Mcginity JW. Stability of Polyethylene Oxide in Matrix Tablets Prepared by Hot-Melt Extrusion. Biomaterials. 2002; 23(21): 4241-4248.

13. Pamu S, Subrahmanyam C, Patnaik K. Hot Melt Extrusion Technique and Its Pharmaceutical Applications: A Review. International Journal of Pharmacy and Analytical Research. 2014; 3(4): 524-530.

14. Lee JY, Kang WS, Piao J, Yoon IS, Kim DD, Cho HJ. Soluplus/TPGS Based Solid Dispersions Prepared by Hot-Melt Extrusion Equipped with Twin-Screw System for Enhancing Oral Bioavailability of Valsartan. Drug des Devel Ther. 2015; 9: 2745-2756.

15. Desai D, Sandhu H, Shah N, Malick W, Zia H, Phauapradit W, Vaka S, Selection of Solid-State Plasticizers as Processing Aids for Hot-Melt Extrusion. J Pharm Sci. 2018: 107: 372-379.

16. Djuris J, Nikolakakis I, Ibric S, Djuric Z, Kachrimanis K. Preparation of CarbamazepineSoluplus Solid Dispersions by Hot-Melt Extrusion and Prediction of Drug Polymer Miscibility by Thermodynamic Model Fitting, Eur J Pharm Biopharm. 2013; 84: 228-237.

17. Douroumis D. Practical Approaches of Taste Masking Technologies in Oral Solid Forms. Expert Opin Drug Del. 2007; 4(4): 417-426.

18. Kokate S, Rachh P. Microparticulate Hot Melt Pellets Technology; A Review. Journal of Drug Delivery and Therapeutics. 2018; 8(6): 377-383.

19. Kolhe S, Chaudhari P, More D. Dissolution Enhancement of Poorly Water Soluble Efavirenz by Hot Melt Extrusion Technique, Int J Drug Dev Res. 2013; 5(2): 368-380.

20. Li L, AbuBaker O, Shao Z. Characterization of Polyethylene Oxide as a Drug Carrier in Hot-Melt Extrusion. Drug Dev Ind Pham. 2006; 32: 9911002.

21. Haung D, Xie Z, Rao Q, Liamas E, Pan P, Gaun S. Hot Melt Extrusion of Heat-Sensitive and High Melting Point Drug: Inhibit the Recrystallization of the Prepared Amorphous Drug During Extrusion to Improve the Bioavailability. Int J Pharm. 2019; 565: 316-324.

22. Huang S, O'Donnell KP, Delpon SM, O'Brien J, Stutzman J, Williams RO. Processing Thermally
Labile Drugs by Hot-Melt Extrusion: The Lesson with Gliclazide. Eur J Pharm Biopharm. 2017; 119: 56-67.

23. Gajda M, Nartowski K, Pluta J, Karolewicz B. The Role of the Polymer Matrix in Solvent-Free Hot Melt Extrusion Continuous Process for Mechanochemical Synthesis of Pharmaceutical Cocrystal. Eur J Pharm Biopharm. 2018; 31 (1): 48-59.

24. McGnity JW, Koleng, Preparation and Evaluation of Rapid Release Granules Using Novel Melt Extrusion Technique. American Association of Pharmaceutical Scientists. 2004; 153-154.

25. Mehuys E, Remon J, Vervaet C. Production of Enteric Capsules by means of Hot-Melt Extrusion. Eur J Pharm Sci. 2005; 24(2): 207-212.

26. Miller D, McConville J, Ynag W, Williams R, McCginity J. Hot-Melt Extrusion for Enhance delivery of drug particles. J Pharm Sci. 2007; 96 (2): 361-376.

27. Morales JO, McConville JT. Manufacture and Characterization of Mucoadhesive Buccal Films. Eur J Pharm Biopharm. 2011;77 (2): 187-199.

28. Repka M, Majumdar S, Battu S, Srirangam R. Upadye S, Applications of Hot-Melt Extrusion for Drug Delivery. Expert Opin Drug Del. 2008; 5: 1357-1376.

29. Repka M. A, Elsohly M. A, Munjal M, Ross S. S. Temperature Stability and Bioadhesive Properties of 9-tetrahydrocannabinol Incorporated Hydroxypropylcellulose Polymer Matrix System, Drug Dev Ind Pharm. 2006; 32 (1): 21-32.

30. Roblegg E, Agar J, Hodzic A, Koscher G, Mohr S, Zimmer A. Development of Sustained-Release Lipophilic Calcium Stearate Pellets via Hot Melt Extrusion. Eur J Pharm Biopharm. 2011; 79(3): 635-645

31. Kolhe S, Chaudhari P, More D. Recent Advances in Hot Melt Extrusion Technology. Int J Pharm Sci Res. 2012; 3(12): 4658-4669.

32. Stankovic M, Frijlink H, Hinrichs W. Polymeric Formulations for Drug Release Prepared by HotMelt Extrusion; Application and Characterization, Drug Discov Today. 2015; 20(7): 812-823.

33. Verreck G, Decorte A, Heymans K. Hot Stage Extrusion of p-amino Salicylic Acid with EC using $\mathrm{CO} 2$ as a Temporary Plasticizer. Int $\mathrm{J}$ Pharm. 2006: 327(1): 45-50.

34. Verreck G, Six K, Van den Mooter G, Baert L, Peeters J, Brewster ME. Characterization of Solid Dispersions of Itraconazole and Hyroxypropylmethylcellulose Prepared by Melt Extrusion. Part I, Int J Pharm. 2003; 251: 165-174.

35. Wedlock D. J, Wijngaarden D. V. Fast Dispersing Solid PVP-Containing Crop Protection Formulation and Process. US patent. 1992, 5: 665,369 .

36. Prodduturi S, Manek RV, Kolling WM, Stodghill SP and Repka M A. "Solid-state stability and characterization of hot-melt extruded poly 
(ethylene oxide) films," Journal of Pharmaceutical Sciences. 2005; 94(10): 2232-2245.

37. Chokshi R J, Shah NH, Sandhu HK, Malick AW and Zia H. "Stabilization of low glass transition temperature indomethacin formulations: impact of polymer-type and its concentration," Journal of Pharmaceutical Sciences. 2008; 97(6): 2286-2298.

38. Rambali B, Verreck G, Baert L, and Massar DL, "Itraconazole formulation studies of the meltextrusion process with mixture design," Drug Development and Industrial Pharmacy, 2003; 29(6): 641-652,

39. De C. Brabander C, Vervaet and J P Remon, "Development and evaluation of sustained release mini-matrices prepared via hot melt extrusion," Journal of Controlled Release. 2003; 89(2): 235247 ,

40. Repka MA, McGinity JW, Zhang F, Koleng JJ. Encyclopedia of pharmaceutical technology, in: J. Boylan (ed.), Marcel Dekker, New York; 2002

41. Lehmann K, Acrylic lattices from redispersable powders for peroral and transdermal drug formulations, Drug Dev. Ind. Pharm. 1986; 12:265-287

42. Amighi $\mathrm{K}$ and Moes AJ. Factors affecting drug release from sustained-release film-coated pellets using acrylic aqueous dispersions, 6th International Conference on Pharmaceutical Technology, Paris, France; 1992.

43. Abdel-Aziz S, Anderson W, The influence of casting solvent composition on structure and permeability of acrylic-methacrylic ester copolymer films, J. Pharm. Pharmac. 1976 28:801805.

44. Zheng X, Yang R, Tang X, Zheng L. Part I: Characterization of Solid Dispersion of Nimodipine Prepared by Hot-Melt Extrusion. Drug Dev Ind Pharm. 2007; 33(7): 791-802.

45. Emara L, Ande; Fattah F, Taha N. Hot Melt Extrusion Method for Preparation of
Ibuprofen/Sucroester WE 15 Solid Dispersions: Evaluation and Stability Assessment, J Appl Pharm Sci. 2017; 7(8): 156-167.

46. Aitken-Nichol C, Zhang F, Mcginity J W. Hot Melt Extrusion of Acrylic Films. Pharm Res. 1996; 13(5): 804-808.

47. J. C. Gutierrez-Rocca and J. W. McGinity, Influence of aging on the physical-mechanical properties of acrylic resin films cast from aqueous dispersions and organic solutions, Drug Dev. Ind. Pharm. 1993 19:315-332.

48. Repka M, Gerding T, Repka S, Mcginity J. Influence of Plasticizer and Drugs on the PhysicalMechanical Properties of Hydroxypropylcellulose Films Prepared by Hot Melt Extrusion. Drug Dev Ind Pharm. 1999; 25(5): 652-633.

49. Schmidt P. C, Niemann F. The MiniWid-Coater Effect of Application Temperature on the Dissolution Profile of Sustained-Released Theophylline Pellets Coated with Eudragit RS30D. Drug Dev Ind Pharm. 1993; 19(13): 1603-1612.

50. Agrawal A, Dudhedia M, Zimny E. Hot melt extrusion; Development of an Amorphous Solid Dispersion for an Insoluble Drug from Mini-Scale to Clinical Scale. AAPS Pharma SciTec. 2016; 17(1): 133-147.

51. Maniruzzaman M, Boateng JS, Chowdhary BZ, Snowden MJ, Douroumis D, A Review on the Taste Masking of Bitter APIs: Hot-Melt Extrusion (HME) Evaluation, J Drug Dev Ind Pharm. 2014; 40(2): $145-56$

52. Aho J, Boetker J, Baldursodottir S, Rantanen J. Rheology as a Tool for Evaluation of Melt Processability of Innovative Dosage Forms, Int J Pharm. 2014; 477: 1-11.

53. Brabander C, Vervaet C, Remon JP. Development and Evaluation of Sustained Release MiniMatrices Prepared via Hot Melt Extrusion. J Control Release. 2003; 89(2): 235-247. 